\title{
A Review on Sensor Network Issues and Robotics
}

\author{
Ji Hyoung Ryu, ${ }^{1}$ Muhammad Irfan, ${ }^{2}$ and Aamir Reyaz ${ }^{1}$ \\ ${ }^{1}$ Chonbuk National University, Jeonju, Republic of Korea \\ ${ }^{2}$ The Infrastructure University Kuala Lumpur (IUKL), Kuala Lumpur, Malaysia \\ Correspondence should be addressed to Aamir Reyaz; aamir8095@gmail.com
}

Received 21 November 2014; Accepted 11 February 2015

Academic Editor: Iftikhar Ahmad

Copyright (C) 2015 Ji Hyoung Ryu et al. This is an open access article distributed under the Creative Commons Attribution License, which permits unrestricted use, distribution, and reproduction in any medium, provided the original work is properly cited.

The interaction of distributed robotics and wireless sensor networks has led to the creation of mobile sensor networks. There has been an increasing interest in building mobile sensor networks and they are the favored class of WSNs in which mobility plays a key role in the execution of an application. More and more researches focus on development of mobile wireless sensor networks (MWSNs) due to its favorable advantages and applications. In WSNs robotics can play a crucial role, and integrating static nodes with mobile robots enhances the capabilities of both types of devices and enables new applications. In this paper we present an overview on mobile sensor networks in robotics and vice versa and robotic sensor network applications.

\section{Introduction}

Technological advances as well as the advent of $4 \mathrm{G}$ communications and of pervasive and ubiquitous computing have promoted a new interest in multihop networks (ad hoc communications). In particular, the interest is in self-organizing wireless multihop networks composed of a possibly large number of motes which can be mobile and static and can also be used for computational and power capabilities. Wireless sensor networks (WSNs) are typical examples of these kinds of networks. Most of the research in WSNs concerns networks whose nodes cannot be replaced and do not move. Mobility of the sensor nodes has been exploited for improving, or enabling altogether, communication coverage and sensing [1]. The credit for the creation of mobile sensor networks goes to wireless sensor networks and to the interaction of distributed robotics. The class of networks where small sensing devices in a collaborative way move in a space to observe and monitor environmental and physical conditions are known as mobile sensor networks [2]. Mobile sensor network is composed of nodes and all nodes have sensing, computation, communication, and locomotion modules (Figure 1). Each sensor node is capable of navigating autonomously or under the control of humans [3]. MSNs have emerged as an important area for research and development.
Though MSNs are still in the developing stages, they can be used for monitoring of environmental habitat, healthcare, agriculture, defense applications, disaster prone areas, hazardous zones, and so forth. MWSNs can also be used for monitored control, and more and more practical applications of MSNs also continue to emerge [4]. And robotics is the science of technology with applications in various fields such as design, fabrication, and theory [5]. It can also be considered as the area of technology dealing with construction, operation, control of robotic applications and computer systems, sensory feedback, and information processing. The main advantage of this technology is that it can replace humans in manufacturing processes and dangerous environments or can also resemble humans in behavior, cognition, or experience [5]. A breakthrough in the autonomous robot technology occurred in the mid-1980s with work in behavior based robotics. We can say that this work was the foundation for many current robotic applications [6]. By incorporating intelligent, mobile robots directly into sensor networks most of the problems in traditional sensor networks may be addressed. Mobile robots offer the ways to interact and survey the environment in a decentralized and dynamic way. The new system of robots and networked sensors led to the development of new solutions to the existing problems such as navigation and localization [7]. Mobile 
nodes can be implemented as autonomous perceptive mobile robots or as perceptive robots whose sensor systems address environmental and navigational tasks. So, we can say robotic sensor networks are the distributed systems in which mobile robots carry sensors around an area to sense phenomena and to produce detailed environmental assessments [8]. The use of multirobot systems for carrying sensors around the environment represents a solution that has received a significant attention and can also provide some extraordinary advantages. A number of applications have been addressed so far by robotic sensor networks, such as rescue, search, and environmental monitoring. In wireless sensor networks, robotics can also be used to solve many problems to advance performances, such as responding to a particular sensor failure, node distribution, and data aggregation. Similarly for solving the problems that exist in the field of robotics wireless sensor networks can play a crucial role. Problems like localization, path planning, coordination for multiple robot, and sensing can be solved by using wireless sensor networks [9]. Today we have many applications of sensor networks on ground, air, underwater, and underground. In mobile UWSN sensor mobility can bring two major benefits. Floating sensors can increase system reusability and can also help to enable dynamic monitoring and coverage. Mobile sensors can help to track changes in water masses thus providing $4 \mathrm{D}$ (space and time) environmental monitoring. As compared to ground based sensor networks mobile UWSNs have to employ acoustic communications because in hard water environments radio does not work. Similarly underground sensor network can be used to monitor a variety of conditions such as properties of soil and environmental monitoring for toxic substances. They are buried completely underground and do not require any wired connections. On the ground they can be used for target tracking, environmental monitoring, detecting forest fire, industrial monitoring, and machine health monitoring. Wireless sensor nodes are into the service from a long time and were being used for different applications such as earthquake measurements and warfare. The recent growth of small sensor nodes dates back to the year 1998 NASA Sensor Webs project and smart dust project. To make autonomous sensing and communication possible within a cubic millimeter of space was the main purpose of the smart dust project. This project led to many more research projects including major research centers in CENS and Berkeley NEST. The term mote was coined by researchers working in these projects to refer to a sensor node; pod is the same term used in the NASA Sensor Webs project for a physical sensor node, although in a Sensor Web the sensor node can be another Sensor Web itself [10].

The main components of a sensor node are as follows: transceiver, a microcontroller external memory, one or more sensors, and power source [10]. The controller processes the data and controls functionality of other components in the sensor nodes. The feasible option of wireless transmission media is infrared, radio frequency (RF), and optical communication. As far as external memory is concerned the most relevant kinds of memory are the flash memory and the on-chip memory of a microcontroller. The most important feature in the development of a wireless sensor node is to

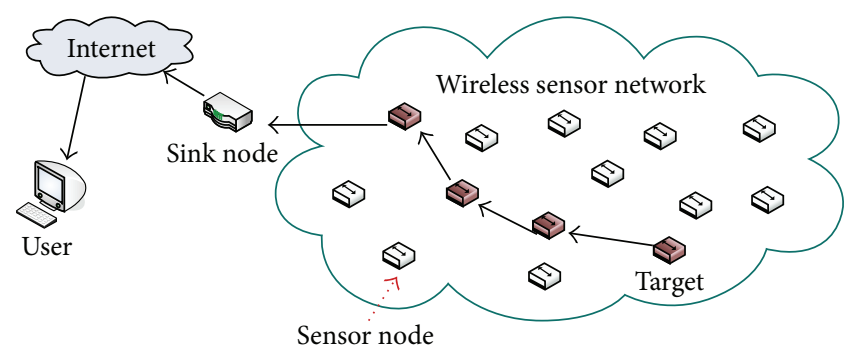

FIGURE 1: Wireless sensor network architecture.

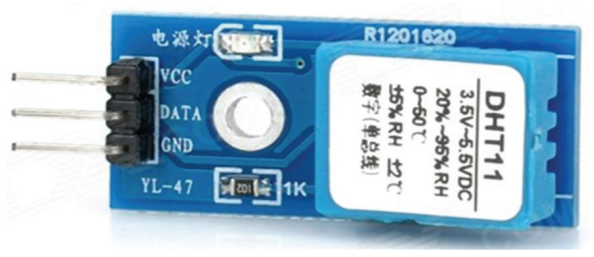

FIgURE 2: Temperature humidity sensor module.

make sure that there is always sufficient energy available to the power system. Sensor nodes consume power for data processing, sensing, and communication; power is stored in capacitors or batteries. Batteries can be both rechargeable and nonrechargeable and for sensor nodes they are the main resource of power supply. And, sensor is a device that detects or senses heat, light, sound, motion, and so forth and then responds to it in a particular way [11] (Figure 2).

The crossbow radio/processor boards usually known as motes permit many sensors scattered over a large area to wirelessly transmit their data back to the base station which is attached to the computer (Figure 3). These motes run TinyOS operating system which is an open source operating system designed for low-power wireless devices, such as those used in PANs, smart meters, ubiquitous computing, sensor networks, and smart buildings [12]. It controls power, radio transmission and networking transparent to the user, and the network which is formed as an ad hoc network [13].

The MICA2 Mote is a third generation mote module with 512 Kbytes of measurement (serial) flash memory, 128 Kbytes of program flash memory, and 4 Kbytes of programmable read-only memory (Figure 4 ).

Stargate is a $400 \mathrm{MHZ}$ Intel PXA255 Xscale processor with 32 Mbytes of flash memory and 64 Mbytes of synchronous dynamic random access memory. A number of classes of sensors are available; these include barometric pressure, acceleration, seismic, acoustic, radar, magnetic camera, light, temperature, relative humidity, magnetic camera, and global positioning system (GPS). Usually sensors are classified into 3 types: passive, omnidirectional, passive, narrow-beam, and active sensors. Passive sensors are selfpowered; they sense the data without actually manipulating the environment by active probing while active sensors actively probe the environment. Narrow beam sensors have a well-defined notion of direction of measurement. Omnidirectional sensors have no notion of direction involved in their measurements [10] (Figure 5). 


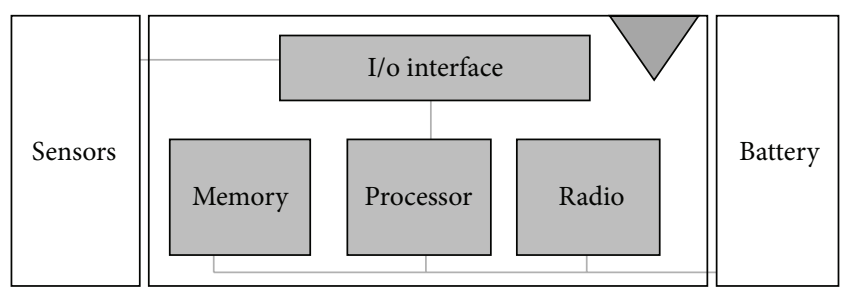

FIGURE 3: A sensor node architecture.

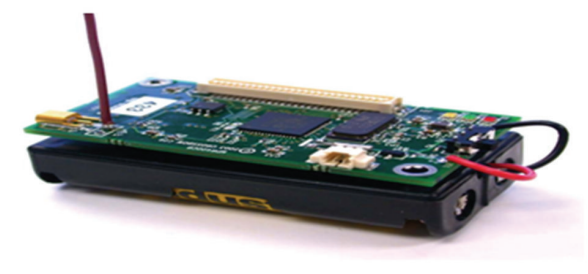

Figure 4: MICA2 processor.

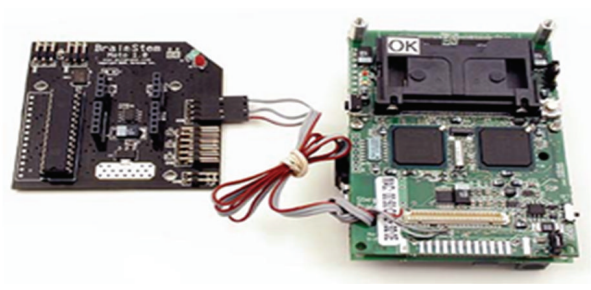

FIGURE 5: Stargate processor.

\section{Mobile Sensor Networks}

Mobile sensor networks are a class of networks where small sensing devices move in a space over time to collaboratively monitor physical and environmental conditions [2]. The research on mobile sensor networks has been plenty worldwide. For MSN, there could be a lot of valuable application with attached sensors as well as capabilities such as locomotion, environmental information sensing, and dead reckoning. The architecture of MSNs can be divided into node, server, and client layer [3]. The job of the node layer is to acquire all sorts of data, as it is directly embedded into physical world. This layer also consists of all the static as well as mobile sensor nodes. Server layer comprises single board computer running server software or a personal computer. The client layer devices can be any smart terminals, these devices also include remote and local clients. Mobility is an unrealistic or undesirable characteristic of sensor nodes as it can address the objective challenges [14]. Research issues on Mobile sensor networks can be analyzed into two aspects [2]: communication issues and data management issues. Our work is focused on communication issues which include coverage and localization issues (Figure 6).

2.1. Coverage. In the sensor networks, coverage can be seen as the measure of quality of service. The quality of surveillance that the network can provide also depends upon the coverage of a sensor network $[15,16]$. It can be seen that, for all the applications of mobile sensor networks, coverage is one of the most fundamental issues [17]. It will decrease due to sensor failure and undesirable sensor deployment. Gage (Gage 92) defines coverage as the maintenance of spatial relationship which adjusts to exact local conditions to optimize the performances of some functions. Gage describes three coverage behavior types. Blanket coverage: its objective is to achieve a static arrangement of nodes that minimizes the total detection area. Barrier coverage: the main goal of the barrier coverage is to reduce the probability of unnoticed penetration through the barrier. Sweep coverage: the concept of sweep coverage is from robotics which is more or less equivalent to moving barrier. The lifetime of sensors is strongly affected by hardware defects, battery depletions, some harsh external environments (e.g., fire, wind), and so forth [2]. In MSNs, previously uncovered areas became covered when sensors move through them and when sensors move away, the already covered areas become uncovered. As a result, the areas covered by sensors change over time, and more areas will be covered at least once as time continues. For robotic applications Khatib [18] was the first one to describe potential field techniques for tasks like local navigation and obstacle avoidance. Similar concept of "motor schemas" was also introduced which uses the superposition of spatial vectors to generate behavior [19]. Howard et al. [20] also used potential fields, but for the deployment problem, they consider the problem of arranging mobile sensors in an unknown environment, where fields are constructed such that each node is repelled by other nodes and also throughout the environment obstacles forces the network to spread. Reference [21] also proposed potential field technique which is distributed and scalable and does not require prior map of the environment. In [22], for the uncovered areas by the sensor network, new nodes are always placed on the boundary of those areas. It is also able to find a suboptimal deployment solution and also makes it sure that each node must be in line of sight with another node. In order to increase the coverage [23] proposed algorithms to calculate the desired target positions where sensors should move and identify the coverage holes existing in the network. To find out the coverage holes Voronoi diagram was used by Wang et al. [24] and he also designed three movement-assisted sensor deployment protocols, namely, VEC (vector based), VOR (Voronoi-based), and Minimax, based on the principle of sensors moving from densely deployed areas to sparsely deployed areas. A virtual force algorithm (VFA) was proposed by [25] to increase sensor field coverage by combining repulsive forces and attractive forces to determine randomly deployed sensors movement and virtual motion paths. Reference [26] deals with both static and mobile sensors and within the sensor field the job is to be served by mobile sensors which appear at random locations. The static sensors then guide the mobile sensors to the position where the task occurs when they get aware about the arrival of tasks. Researchers deal with the dynamic aspects of coverage in mobile sensor networks and also characterized area coverage at specific time instants and during time interval and detection time of the randomly located target [27]. The problem of coverage and exploration 


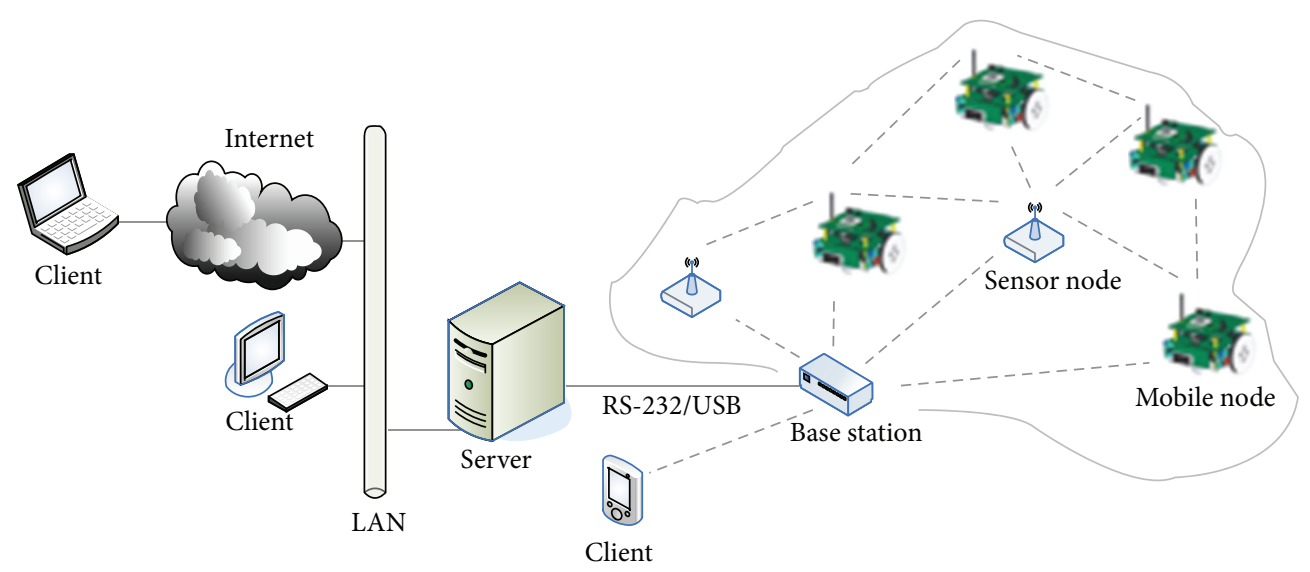

FIGURE 6: The system architecture of a mobile sensor network.

through the utilization of deployed network was considered and the algorithm which assumes that the global information is not available was also presented [28]. In [29] the problem of sensor relocation is being focused and two-phase sensor relocation solution has been proposed in which redundant sensors are identified first using Grid-Quorum and then are relocated in a cascaded movement in a timely, efficient, and balanced way [2].

2.2. Localization. Recently, there has been much focus on building mobile sensors, and we have seen the development of small-profile sensing devices that are quite capable of controlling their own movement. Mobility has become an important area of research for mobile sensor networks. Mobility enables sensor nodes to target and track moving phenomena such as vehicles, chemical clouds, and packages [30]. One of the most significant challenges for mobile sensor nodes is the need for localization. Localization is the ability of sensor nodes to find out its physical coordinates, and localization on mobile sensors is performed for navigational and tracking purposes. Localization is required in many applications in wireless sensor networks such as health, military, and industry. Extensive research has been done so far on localization. Many location discovery schemes have proposed to eliminate the need of GPS on every sensor node [31]. GPS is commonly considered to be a good solution for outdoor localization. However, GPS is still expensive and hence insufficient to be used for large number of devices in WSN. Some of the problems with GPS are as follows.

These are some situations in which GPS will not work reliably because GPS receiver needs line of sight to the multiple satellites and it does not work well in the indoor environment. And GPS receivers are available for mote scale devices only. They are still expensive and undesirable for many applications. Even if GPS receivers became cheaper and are used in every node, the nodes cannot actively use GPS in mobile sensor networks. Typically GPS node consumes more energy than sensors and low-power transceivers. The problem of using GPS in a real environment also exists in GPS itself. GPS shows 10 20 m of error when used in normal outdoor environments unless it uses a costly mechanism such as differential GPS. Deploying a large number of GPS in mobile sensor network has both limits and possibilities [32]. There are two types of localization algorithms, namely, centralized and distributed algorithms [31]. These centralized location techniques depend on sensor nodes transmitting data to a central location, where computation is performed to find out the location of each node [33]. Distributed algorithms do not need a central base station and for determining its location it relays on each node with only limited communication with nearby nodes [31]. Localization algorithms in MWSNs can be categorized into (1) range-based method, (2) range-free method, (3) mobility based method [2]. All of these methods vary in the information used for localization purposes. Range-based methods use range measurements while rangefree techniques only use the content of messages [34]. Rangebased methods also require expensive hardware to measure signal arrival time and angle of signal arrival. As compared to range-free methods these methods are expensive because of their pricey hardware [2]. Range-based approaches have also utilized time of arrival, received signal strength, time difference of arrival of two different signals (TDOA), and the angle of arrival. Though they can reach the fine resolution, either the required hardware is expensive or the results depend on impractical assumptions about signal propagation [33]. While range-free methods use local and hop count techniques, for range-based approaches these methods are very cost effective. Many localization algorithms have been proposed so far such as elastic localization algorithm (ELA) and mobile geographic distributed localization algorithm; both of these algorithms assume nonlimited storage in sensor nodes [2]. For sensor networks [33] two types of range-free algorithms have been proposed: local techniques and hop count techniques. Local techniques rely on high speed on a high density of seeds so that every node can hear several seeds and hop count techniques rely on a flooding network. Each node in centroid method estimates its location by calculating the center of the seeds locations it hears. Location error can be reduced if seeds are well positioned but in ad hoc deployments this is impossible. The APIT method separates the environment into triangular regions between beaconing nodes and to calculate the maximum area it uses the grid 

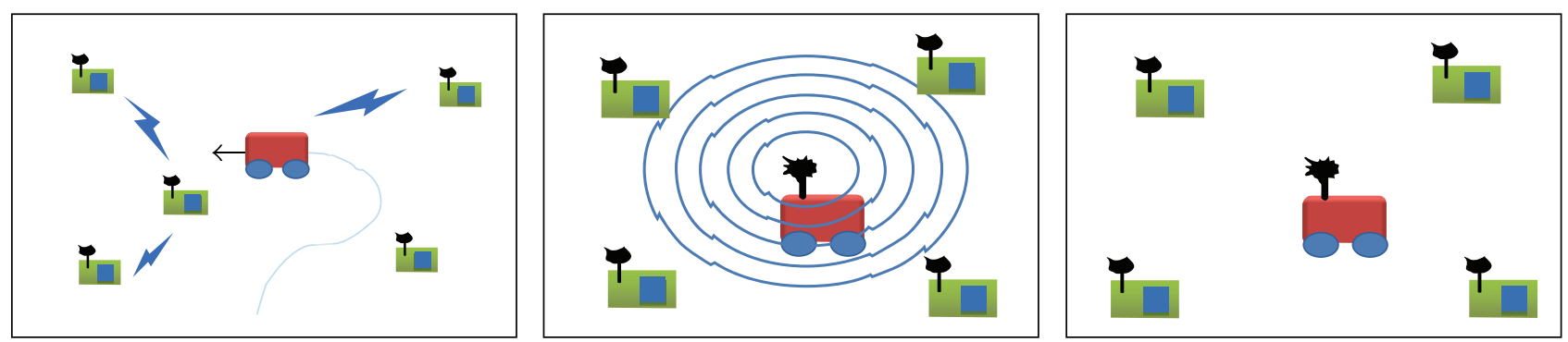

FIgURE 7: Coordination, measurement, and location estimation phase.

algorithm in which a node will likely reside [33]. Hop count techniques propagate the location estimation throughout the network where the seed density is low. In mobility-based methods, to improve accuracy and precision of localization method sequential Monte Carlo localization (SML) was proposed [27] without additional hardware except for GPS [2]. And without decreasing the nonlimited computational ability many techniques using SML are also being proposed. In order to achieve the accurate localization researchers proposed many algorithms, using the principles of Doppler shift and radio interferometry to achieve the accurate localization has also been used [2]. The three phases typically used in localization are (1) Coordination, (2) measurement, and (3) position estimation [30] (Figure 7).

To initiate the localization a group of nodes coordinate first, a signal is then emitted by some nodes, and then some property of the signal is observed by some other nodes. By transforming the signal measurements into position estimates node position is then determined. To find the positions of sensors in order to reduce the frequency of localization, three techniques were proposed: static fixed rate (SFR), dynamic velocity monotonic (DVM) and mobility aware dead reckoning driven (MADRD) [35].

(1) Static fixed rate (SFR): the performance of this protocol varies with the mobility of sensors. In this base protocol each sensor invokes its localization periodically with a fixed time period $t_{s} f_{r}$. In this technique, the error will be high if the sensor is moving quickly and if it is moving slowly the error will be low [36]:

$$
e_{s f r}=\frac{n \times \sin \theta}{\sin \alpha}
$$

(2) Dynamic velocity monotonic: this is an adaptive protocol; with the mobility of sensors localization is called adaptively in DVM; the higher the observed velocity is, the faster the node should localize to maintain the same level of error. It computes the node velocity when it localizes by dividing the distance it has moved since the last localization point by the time that elapsed since localization. The next localization point is scheduled based on the velocity at the time when a prespecified distance will be travelled if the node continues with the same velocity [37].
(3) Mobility aware dead reckoning driven (MADRD): to predict the future mobility this protocol computes the mobility pattern of the sensors. When the expected difference between the predicted mobility and expected mobility reaches the error threshold the localization should be triggered [38]:

$$
\operatorname{Err}_{\text {madrd }}=\int_{0}^{T} E\left[\left(x-x^{\prime}\right)^{2}+\left(y+y^{\prime}\right)^{2}\right] d t .
$$

Mobility aware interpolation (MAINT) was proposed to estimate the current position with better trade-off between energy consumption and accuracy [37]. Their method uses interpolation which gives better estimation in most cases:

$$
\operatorname{Err}_{\text {maint }}=\int_{0}^{T} E\left[\left(x-x^{\prime \prime}\right)^{2}+\left(y+y^{\prime \prime}\right)^{2}\right] d t
$$

2.3. Positioning Systems. Many methods have been used for the problem of localization. Positioning systems will use positioning technology to determine the position and orientation of an object or person in a room [39].

2.3.1. Xbee Technology. It is a brand of radios that support a variety of communication protocols. It uses Zigbee protocol; Zigbee is a wireless communication protocol like $\mathrm{Wi}-\mathrm{Fi}$ and Bluetooth. These modules use the IEEE 802.15.4 networking protocol for fast point-to-multipoint or peer-to-peer networking. Its low-power consumption limits transmission distances to 10-100-meter line of sight though, depending on power output and environmental characteristics [40]. It operates in unlicensed ISM bands, so it is prone to interference from a wide range of signal types using the same frequency which can disrupt the radio frequency. From RSSI values the distance between two Zigbee nodes is calculated; based on the distance calculated from Zigbee modules many researchers found it suitable for indoor localization. It comes with modules, though orientation is the problem in many modules because most of its modules are without omnidirectional antenna (Figure 8).

2.3.2. Wi-Fi Based Indoor Localization. Wi-Fi, or wireless networking, is one of the biggest changes to the way we use computers since the PC was introduced. Wi-Fi also allows communications directly from one computer to another 


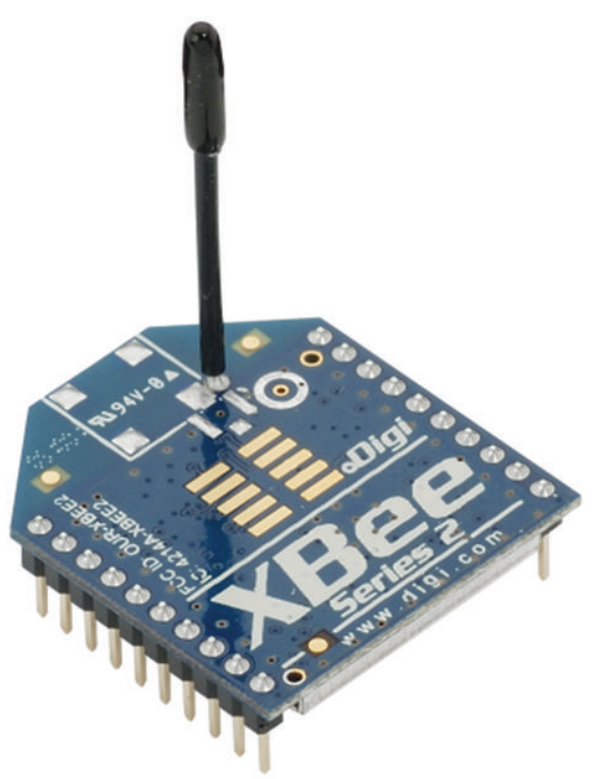

Figure 8: Xbee.

without an access point intermediary. This is called ad hoc Wi-Fi transmission. A typical wireless access point using 802.11 b or $802.11 \mathrm{~g}$ with a stock antenna might have a range

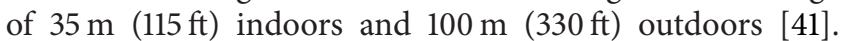
The widespread availability of wireless networks (Wi-Fi) has created an increased interest in harnessing them for other purposes, such as localizing mobile devices. There has long been interest in the ability to determine the physical location of a device given only Wi-Fi signal strength. This problem is called Wi-Fi localization and has important applications in activity recognition, robotics, and surveillance. The key challenge of localization is overcoming the unpredictability of Wi-Fi signal propagation through indoor environments. The data distribution may vary based on changes in temperature and humidity, as well as position of moving obstacles, such as people walking throughout the building. The uncertainty makes it difficult to generate accurate estimates of signal strength measurements. Wi-Fi based systems have number of issues, including high power consumption, being limited to coverage, and being prone to interference [42].

2.3.3. Ultrawideband and FM Radio Based Technique. To achieve high bandwidth connections with low-power consumption ultrawideband is the best communication method. Ultrawideband wireless radios send short signal pulses over broad spectrum [43]. This technology has been used in a variety of localization tasks requiring higher accuracy $20-30 \mathrm{~cm}$ than achievable through conventional wireless technologies, for example, radio frequency identification (RFID), WLAN, and so forth [41]. The limitation with this technique is that it requires specialized hardware and dedicated infrastructure, resulting in high costs for wide adoption [42]. FM radios can be used for indoor localization, while providing longer battery life than Wi-Fi, making FM an alternative to consider for positioning. FM radio signals are less affected by weather conditions such as rain and fog in comparison to Wi-Fi or GSM. These signals penetrate walls easily as compared to $\mathrm{Wi}-$ $\mathrm{Fi}$; this makes sure high availability of FM positioning signals in indoor environments. FM radio uses frequency division multiple access (FDMA) approach which splits the band into number of frequency channels that are used by stations. There are only few papers dedicated to FM radio based positioning.

2.3.4. Bluetooth Positioning System. By executing the inquiry protocol Bluetooth device detects other devices. Devices within its range that are set to "discoverable" will respond by identifying themselves. Bluetooth communicates using radio waves with frequencies between $2.402 \mathrm{GHz}$ and $2.480 \mathrm{GHz}$, which is within the $2.4 \mathrm{GHz}$ ISM frequency band, a frequency band that has been set aside for industrial, scientific, and medical devices by international agreement. The main advantage of using Bluetooth is that this technology is of high security, low power, low cost, and small size. Many researchers have used Bluetooth for indoor positioning and this reference used Bluetooth [44].

2.3.5. Radio Frequency Identification. An RFID (radio frequency identification) system consists of a reader with an antenna which interrogates nearby active transceivers or passive tags. Using RFID technology data can be transmitted from RFID tags to the reader via radio waves. The data consists of the tags unique ID (i.e., its serial number) which can be related to available position information of the RFID tag. These are used in localization because of their advantages; radio waves can pass through walls, obstacles, and human bodies easily. This technique needs less hardware and has large coverage area. By using advanced identification technology and noncontact, this technology uses one way one wireless communication that uses radio signals to put an RFID tag on objects and people to track them and automatically identify them [41].

2.3.6. Hybrid Positioning System. These systems use several different technologies to find the location of a mobile device by using many positioning technologies. To overcome the limitations of GPS, these systems are mainly developed because GPS does not work well in indoors. This system is being highly investigated for commercial and civilian based location services like Google maps for mobile, devicescape, and so forth $[41,45]$.

2.3.7. Quick Response Code. It is a matrix code that keeps a comparative huge amount of location information compared to standard barcode. It can be attached to some key areas of the buildings to wait for scanning to provide its positional information from database [46].

In this section we present a brief overview of the localization methods used by some researchers. In localization using RSSI, based on Xbee modules in wireless sensor networks, [47] proposed a localization technique using RSSI, and the technique is based on decision tree obtained from a set of empirical experiments. By applying the Cramer's rule approach they used the decision tree to select the best three 
neighbor reference nodes that are involved in the estimation of the position of target sensor node. The results obtained based on empirical data and gathered from the experiments indicate accuracy less than 2 meters. By using Zigbee CC2431 modules [48] proposed closer tracking algorithm for indoor wireless sensor localization. The proposed algorithm can suitably select an adaptive mode to obtain precise locations. They also improved the fingerprinting algorithm in mean time. The proposed technique CTA can determine the position with error less than 1 meter. Based on artificial neural networks [49] presented location estimation system in the indoor environment. This architecture provides robust mechanism for coping with unavailable information in real life situations as they employ modular multilayer perceptron (MMLP) approach to effectively reduce the uncertainty in the location estimation system. Moreover their system does not require runtime searching of nearest neighbors in huge backend database. Yu proposed a measurement and simulation based work of a fingerprinting technique based on neural networks and ultrawideband signals. Their proposed technique is based on the construction of a fingerprinting database of LDPs extracted from an UWB measurement campaign. To learn the database and to locate the targeted positions the feed forward neural network with incremental back backpropagation is used. In order to evaluate the positioning performance, different types of fingerprinting database and different sizes are considered [50]. To perform indoor localization, [51] evaluated several ANN designs by exploiting RSS fingerprints collected in an office environment. They relied on WLAN infrastructure to minimize the deployment cost. The proposed cRBF algorithm can be a good solution to the location estimation problem in indoor environments. Moreover based on their experimental results, it is found that the proposed algorithm achieves more accuracy compared to sRBF, MLP and GRNN designs, and KNN algorithm. Reference [52] used RSS fingerprinting technique and artificial neural network for mobile station location in the indoor environment. The proposed system learns offline the location "signatures" from extracted location-dependent features of the measured data for LOS and NLOS situations and then it matches online the observation received from a mobile station against the learned set of "signatures" to accurately determine its position. It was found that location precision of the proposed system is 0.5 meters for $90 \%$ of trained data and 5 meters for $45 \%$ of untrained data. By using RSSI values of anchor node beacons, researchers presented an artificial feed forward neural network based approach for node localization in sensor networks. They evaluated five different training algorithms to obtain the algorithm that gives best results. The multilayer perceptron (MLP) neural network has been obtained using the Matlab software and implemented using the Arduino programming language on the mobile node to evaluate its performance in real time environment. By using 12-12-2 feed forward neural network structure, an average error of $30 \mathrm{~cm}$ is obtained [53]. To infer the clients position in the wireless local area network (LAN), researchers presented a novel localization algorithm, namely discriminant adaptive neural network (DANN), which takes, received signal strength (RSS) from access points (APs) as an input. For network learning, they extracted the useful information into discriminative components (DCs). This approach incrementally inserts DCs and recursively updates the weightings in the network until no further improvement is required. Traditional approaches were implemented on the same test bed, including weighted-nearest neighbor (WKNN), maximum likelihood (ML), and multilayer perceptron (MLP), and then results were compared. The results showed that the proposed technique has better results as compared to other examined techniques [54]. Ali used neural networks to solve the problem of localization in sensor networks. They compared the performances of three different families of neural networks: multilayer perceptron (MLP), radial basis function (RBF), and recurrent neural networks (RNN). They compared these networks with two variants of Kalman filter which are also used for localization. Resource requirements in terms of computational and memory resources were also compared. The experimental results in [55] show that RBF neural network has the best performance in terms of accuracy and MLP neural networks has best computational and memory resource requirement. Another neural network based approach was used by Laslo. For the processing, received signal strength indicator (RSSI) was used and its also used for learning of neural network and preprocessed (mean, median and standard deviation) in order to increase the accuracy of the system. Fingerprint (FP) localization methodology was also applied in the indoor experimental environment which is also presented. The RSSI values used for the learning of the neural network are preprocessed (mean, median, and standard deviation) in order to increase the accuracy of the system. To determine the accuracy of the neural network, mean square error of Euclidean distance between calculated and real coordinates and the histogram was used in [56]. Wi-Fi based technique was proposed for detecting users position in an indoor environment. They implemented the trilateration technique for localization. They used mobile phone to obtain RSSI from the access points and then the RSSI data was converted into distance between users and each AP. They also proposed to determine the users position based on trilateration technique [57]:

$$
\text { distance, } d_{i}=p\left(1-m_{i}\right) \text {, }
$$

where $m$ is the percentage of signal strength, $p$ is the maximum coverage of signal strength, and $I=1,2,3$. By analyzing a large number of experimental data, it is found that the variance of RSSI value changes along with the distance regularly. They proposed the relationship function of the variance of RSSI and distance and establish the lognormal shadowing model with dynamic variance LNSM-DV based on the result analysis. Results show that LNSM-DV can further reduce error and have strong self-adaptability to various environments compared with LNSM [58]. In this study the problem of indoor localization using wireless Ethernet IEEE 802.11 (Wireless Fidelity, Wi-Fi) was analyzed. The main purpose of this work was to examine several aspects of location fingerprinting based on indoor localization that affects positioning accuracy. The results showed that they achieved the accuracy of 2-2.5 meters [59]. In the mobile 
node localization, a system was proposed for real environment when both anchors and unknown nodes are moving. To figure out the current position, history of anchor information was used. User's movement was modeled by the archived information and for discovering new positions movement models were also used. In complex situations where anchors and nodes are mobile, researchers presented three methods to resolve localization problem [38]. The proposed methods take into account the capability of nodes: nodes which can calculate either distances or angles with their neighbors or none of both. When the sensor has at least two anchors in the neighborhood, the proposed methods determine the exact position; else it gives a fairly accurate position and in this case it can compute the generated maximal error. The proposed method also defines periods when a node has to invoke its localization. A GPS free localization algorithm was in MWSNs [60]. In order to build the coordinate system, the proposed algorithm uses the distance between the nodes and also nodes positions are computed in two dimensions. Based on dead reckoning, Tilak et al. [36] proposed a number of techniques for tracking mobile sensors. Among all the proposed techniques, mobility aware dead reckoning estimates the position of the sensor, instead of localizing the sensor every time it moves. Error in the estimated position is calculated every time the localization is called and with the time error in the estimation grows. And also the next localization time is fixed depending on the value of this error. For a given level of accuracy in position estimation fast mobile sensors trigger localization with higher frequency. Instead of localizing sensor, a technique was proposed to estimate the positions of a mobile sensor [37]. It gives higher accuracy for particular energy cost and vice versa. When sensor has some data to be sent, the position of the sensor is required then only. The information of an inactive sensor is ceased to be communicated. In order to reduce the arithmetic complexity of sensors, most calculations are carried out at base station. To solve the set of equations, [31] proposed the novel algorithm for MSN localization in which they took three nodes which are neighbors to each other and if the solutions are unique they are the node positions. And, for searching the position of the final node, a scan algorithm was also introduced called a metric average localization error (ALE) (which is the root mean square error of node locations divided by the total number of nodes) to evaluate the localization error:

$$
\mathrm{ALE}=\frac{\sum_{i=1}^{N}\left\|p_{i}-K_{i}\right\|}{N} .
$$

\section{Security Issues in Mobile Ad Hoc Sensor Networks}

Because of the vulnerability of wireless links, nodes limited physical protection, nonexistence of certification authority, and lack of management point or a centralized monitoring, it is difficult to achieve security in mobile ad hoc networks [61]. Sensor networks have many applications: they are used in ecological, military, and health related areas. These applications often examine some sensitive information such as location detection or enemy movement on the battlefields. Therefore security is very important in WSN. WSN has many limitations such as small memory, limited energy resources, and use of insecure channels in communication; these problems make security in WSN a challenge [62]. Designing the security schemes of wireless sensor networks is not an easy task; sensor networks have many constraints compared to computer networks [63]. The main objective of the security service in WSN is to protect the valuable information and resources from misbehavior and attacks; requirements in wireless sensor network security include availability, authorization, confidentiality, authentication, integrity, nonrepudiation, and freshness. Attacks in WSN can be categorized as follows: attacks on network availability: these are often referred to as DOS (denial of service attacks); these attacks can target any layer of a sensor network. Attacks on secrecy and authentication include packet replay attacks, eavesdropping, or spoofing of attacks. There are also stealthy attacks against service integrity. In this type of attack the motive of the attacker is to make network accept false data value. Security threats and issues in WSN can be classified into two categories: active and passive attacks [63].

3.1. Active Attacks. It implies the disruption of the normal functionality of the network, meaning information interruption, modification, or fabrication [64]. Impersonating, modification, fabrication, message replay, and jamming are the examples of active attacks. The following attacks are active in nature.

3.2. Routing Attacks in Sensor Networks. Routing attacks are the attacks which act on the network layer. While routing the messages many attacks can happen; some of them are as follows: attacks on information in transit, selective forwarding, and Blackhole/Sinkhole attack.

3.3. Wormhole Attacks. In this type of attack, the attacker records packets at one location in the network and then tunnels them to another location and retransmits them there into the network [65].

3.4. HELLO Flood Attacks. In this type of attack the attacker uses HELLO packets to convince the sensors in WSN. The attacker sends routing protocols HELLO packets from one node to another with more energy [63].

3.5. Denial of Service (DOS). These attacks can target any layer of a sensor network. This type of service is produced by unintentional failure of nodes or malicious action.

3.6. Node Subversion. Capture of a node may disclose its information and thus compromise the whole sensor network. In this attack the information stored on sensor might be obtained by attacking it [66].

3.7. Node Outage. When a node stops its function, this kind of situation occurs. In the case where a cluster leader stops 
functioning, the sensor network protocols should be robust enough to mitigate the effects of node outages by providing an alternate route.

3.8. Node Malfunction. It can expose the integrity of a sensor network if the node starts malfunctioning.

3.9. Physical Attacks. These types of attacks can destroy sensors permanently. These attacks on sensor networks typically operate in hostile outdoor environments. Attackers can extract cryptographic secrets, modify programming in the sensors, tamper with associated circuitry, and so forth.

3.10. False Node. This can lead to injection of malicious data by the additional node; this can spread to all the nodes, potentially destroying the whole network. In this case an intruder adds a node to the system that feeds false data or it can also prevent the passage of true data.

3.11. Massage Corruption. Any change in the content of a message by an attacker compromises its integrity [63].

3.12. Node Replication Attacks. In this attack, attacker adds an additional node to the existing network by copying the node ID. This can result in a disconnected sensor network and false sensor readings.

3.13. Passive Information Gathering. Strong encryption techniques need to be used in order to minimize the threats of passive information gathering [63].

3.14. Passive Attacks. In passive attacks, the attack obtains data exchange in the network without interrupting the communication [64]. Some of the most common attacks against sensor privacy are as the following.

3.14.1. Monitoring and Eavesdropping. The most common attack to the privacy is the monitor and eavesdropping. Eavesdropping is the intercepting and reading of messages and conversations by unintended users [64].

3.14.2. Traffic Analysis. There is a high possibility analysis of communication patterns even when the messages transferred are encrypted. The activities of sensor can disclose a lot of information to enable an adversary to cause malicious harm to the network.

3.14.3. Camouflage Adversaries. In the network one can comprise the nodes to hide or can insert their node. After inserting their node, that node can work as a normal node to attract packets and then misroute them which can affect privacy analysis.

\section{Robotic Sensor Network Applications}

Most of the problems in traditional sensor networks may be addressed by incorporating intelligent, mobile robots directly into it. Mobile robots provide the means to explore and interact with the environment in a dynamic and decentralized way. In addition to enabling mission capabilities well beyond those provided by sensor networks, these new systems of networked sensors and robots allow for the development of new solutions to classical problems such as localization and navigation [1]. Many problems in sensor networks can be solved when putting robotics into use, problems like node positioning and localization, acting as data mule, detecting and reacting to sensor failure, for nodes mobile battery chargers, and so forth. And also wireless sensor networks can help solve many problems in robotics such as robot path planning, localization mapping, and sensing in robots [9]. Mobile nodes can be implemented as autonomous perceptive mobile robots or as perceptive robots whose sensor systems address environmental task and navigational task. Robotic sensor networks are particular mobile sensor networks or we can say robotic sensor networks are distributed systems in which mobile robots carry sensors around an environment to sense phenomena and to produce in depth environmental assessments. There are many applications of wireless sensor networks in robotics like robotics advanced sensing, coordination in robots, robot path planning, and robot localization, robot navigation, network coverage, proper data communication, data collection, and so forth. Using WSN helps emergency response robots to be conscious of the conditions such as electromagnetic field monitoring and forest fire detection. These networks improve the sensing capability and can also help robot in finding the way to the area of interest. WSNs can be helpful for coordinating multiple robots and swarm robotics because the network can assist the swarm to share sensor data, tracking its members, and so forth. To perform the coordinated tasks it sends robots to the different locations and also a swarm takes decisions based on the localization of events, allowing path planning and coordination for multiple robots to happen efficiently and optimally and direct the swarm members to the area of interest. In the localization part there are many techniques for localizing robots within a sensor network. Cameras have been put into use to identify the sensors equipped with infrared light to triangulate themselves based on distances derived from pixel size. A modified SLAM algorithm has been utilized by some methods which uses robots to localize itself within the environment and then compensates for SLAM sensor error by fusing the estimated location with the estimated location in WSN based on RSSI triangulation [9]. An intruder detection system was presented in [59] which uses both wireless sensor networks and robots. In order to learn and detect intruders in previously unknown environment, sensor network uses an unsupervised fuzzy adaptive resonance theory (ART) neural network. A mobile robot travels upon the detection of an intruder to the position where the intruder is detected. The wireless sensor network uses a hierarchical communication/learning structure where mobile robot is root node of the tree.

In wireless sensor networks robotics can also play a crucial role. They can be used for replacing broken nodes, repositioning nodes, recharging batteries, and so forth. To increase the feasibility of WSNs, [67] used robots because 
they have actuation but limited coverage in sensing while sensor networks lack actuation but they can acquire data. In servicing WSNs, robot task allocation and robot task fulfillment was examined [68]. Problems are examined in robot task allocation such as using multitask or single-task robots in a network and how to organize their behavior to optimally service the network. The route which a robot takes to service nodes is examined in robot task fulfillment. To improve the robot localization, [69] adapts sensor network models with information maps and then checks the capability of such maps to improve the localization. The node replacement application was developed by [70] in which a robot would navigate a sensor network based on RSSI (received signal strength indication) from nearby nodes and it would then send a help signal if a mote will begin to run low on power. And, then through the network, the help signal would be passed to direct the robot to replace the node. Robots can also be used to recharge batteries. The problem of localization can also be solved using robots; they can be used to localize the nodes in the network. They can also be used in data aggregation. In a network, they can also serve as data mules; data mules are robots that move around the sensor network to collect data from the nodes and then transport that data back to sink node or perform the aggregation operations operation on data [9]. The use of multirobot systems for carrying sensors around the environment represents a solution that has received a considerable attention and can provide some remarkable advantages as well. A number of applications have been addressed so far by robotic sensor networks, including environmental monitoring and search and rescue. When put into use robotics sensor networks can be used for effective search and rescue, monitoring electromagnetic fields, and so forth. Search and rescue systems should quickly and accurately locate victims and map search space and locations of victims and with human responders it should maintain communication. For a search and rescue system to fulfill its mission, the system should be proficient to rapidly and reliably trace its victims within the search space and should also be well capable to handle a dynamic and potentially hostile environment. For utilizing ad hoc networks, [7] presented an algorithmic framework consisting of a large number of robots and small, cheap, simple wireless sensors to perform proficient and robust target tracking. Without dependence on magnetic compass or GPS localization service, they described a robotic sensor network for target tracking, focusing on algorithms which are simple for information propagation and distributed decision making. They presented a robotic sensor network system that autonomously conducts target tracking without component possessing localization capabilities. The approach provides a way out with minimal hardware assumptions (in terms of sensing, localization, broadcast, and memory/processing capabilities) while subject to a dynamic changing environment. Moreover the framework adjusts dynamically to both target movement and addition/deletion of network components. The network gradient algorithm provides an advantageous trade-off between power consumption and performance and requires relatively bandwidth [71]. The monitoring of EMF phenomena is extremely important in practice, especially to guarantee the protection of the people living and working where these phenomena are significant. A specific robotic sensor network oriented to monitor electromagnetic fields (EMFs) was presented [8]. The activities of the system are being supervised by a coordinator computer, while a number of explorers (mobile robots equipped with EMF sensors) navigate in the environment and perform EMF measurement tasks. The system is a robotic sensor network that can autonomously deploy its explorers in an environment to cope with events like a moving EMF source. The system architecture is hierarchical. The activities of the system are being supervised by the computer and to perform the EMF tasks a number of explorers (mobile robots equipped with EMF sensors navigate through the environment). The grid map of the environment is maintained by the system, in which each cell can be either free or occupied by an obstacle or by a robot. The map is supposed to be known by the coordinator and the explorers. The environment is assumed to be static and the map is used by the explorers to navigate in the environment and by the coordinator to localize the EMF source [72].

\section{Coverage for Multirobots}

The use of multirobots holds numerous advantages over a single robot system. Their potential of doing work is way far better than that of single robot system [73]. Multiple robots can increase the robustness and the flexibility of the system by taking benefits of redundancy and inherent parallelism. They can also cover an area more quickly than a single robot and they have also potential to accomplish a single task faster than a single robot. Multiple robots can also localize themselves more efficiently when they have different sensor capabilities. Coverage for multirobot systems is an important field and is vital for many tasks like search and rescue, intrusion detection, sensor deployment, harvesting and mine clearing, and so forth [74]. To get the coverage the robots must be capable of spotting the obstacles in the environment and they should also exchange their knowledge of environment and have a mechanism to assign the coverage tasks among themselves [75]. The problem of deploying a mobile senor network into an environment was addressed in [76] with the task of maximizing sensor coverage and also two behavior based techniques for solving the 2D coverage problems using multiple robots were proposed. Informative and molecular techniques are the techniques proposed for solving coverage problems and both of these techniques have the same architecture. When robots are within the sensor range of each other, the informative approach is to assign local identities to them. This approach allows robots to spread out in a coordinated manner because it is based on ephemeral identification where temporary local identities are assigned and mutual local information is exchanged. No local identification is made in molecular approach and also robots do not perform any directed communication. Each robot moves in a direction without communicating its neighbors because it selects its direction away from all its immediate sensed neighbors. Then these algorithms were compared with another approach known as basic approach, which only seeks 
to maximize each individual robot's sensor coverage [74]. Both these approaches perform significantly better than basic approach and with the addition of few robots the coverage area quickly maximizes. An algorithm named (StiCo) which is an coverage algorithm was proposed for multirobot systems in [77]. This algorithm is based on the principle of stigmergic (pheromone-type) coordination known from the ant societies where a group of robots coordinate indirectly via ant-like stigmergic communication. This algorithm does not require any prior information about the environment and also no direct robot-robot communication is required. Similar kind of approach was used by Wagner et al. [78] for coverage in multirobot in which a robot deposits a pheromone which could then be detected by other robots; these pheromones come up with a decay rate, allowing continuous coverage of an area via implicit coordination [75]. For multirobot coverage, [75] proposed boustrophedon decomposition algorithm in which the robots are initially distributed through space and each robot is allocated at virtually bounded area to cover the area and is then decomposed into cells with the fixed cell width. By using the adjacency graph the decomposed area is represented which is incrementally constructed and shared among all robots and without any restriction robot communication is also available. By sharing information regularly and task selection protocol performance is improved. By planting laser beacons in environment the problem of localization in the hardware experiment was overthrown and using the laser range finder to localize the robots as this was the major problem to guarantee accurate and consistent coverage. Based on spanning-tree coverage of approximate cell decomposition, robustness and efficiency in a family of multirobot coverage algorithms was addressed [79]. Their approach is based on offline coverage: it is assumed that the robots have a map of the area a priori. The algorithm they proposed decomposes the work area into cells, where each cell is a square of size $4 \mathrm{D}$ and each cell is then further broken down into quadrants of size D.

\section{Localization for Robots}

In mobile robotics localization is a key component [80]. The process to determine the robots position within the environment is called localization or we can say that it is a process that takes a map as an input and estimates the current pose of the robot, a set of sensor readings, and then outputs the robot's current pose as a new estimate [81]. There are many technologies available for robot localization including GPS, active/passive beacons, odometer (dead reckoning), and sonar. For robot localization and map count an algorithm was presented using data from a range based sonar sensor [82]. For localization the robots position is determined by the algorithm by correlating a local map with a global map. Actually no prior knowledge of the environment is assumed; it uses sensor data to construct the global map dynamically. The algorithm estimates robots location by computing positions called feasible poses where the expected view of robot matches approximately the observed range sensor data. The algorithm then selects the best fit from the feasible poses. It requires robots orientation information to make sure that the algorithm identifies the feasible poses. For location information Vassilis also used dead reckoning as a secondary source; when combined with range sensor based localization algorithm it can provide a close real time location estimate. A Monte Carlo localization algorithm was introduced using (MHL) was used for mobile robot position estimation [83]. They used the Monte Carlo type methods and then combined the advantages of their previous work in which grid based Markov localization with efficiency and accuracy of Kalman filter based techniques was used. MCL method is able to deal with ambiguities and thus can globally localize the robot. As compared to their previous grid based method MCL method has significantly reduced memory requirements while at the same time incorporating sensor measurements at a considerably higher frequency. Based on condensation algorithm the Monte Carlo localization method was proposed in [84]. It localizes the robot globally using a scalar brightness measurement when given a visual map of the ceiling. Sensor information of low feature is used by these probabilistic methods specifically in $2 \mathrm{D}$ plane and needs the robot to move around for probabilities to gradually converge toward a pack. The pose of the robots was also computed by some researchers based on the appearance. Panoramic image-based model for robot localization was used by Cobzas and Zhang [55]; with the depth and 3D planarity information the panoramic model was constructed, while the matching is based on planar patches. For probabilistic appearance based robot localization [56] used panoramic images. For extracting the 15-dimensional feature vectors for Markov localization PCA is applied to hundreds of training images. In urban environments the problem of mobile robot localization was addressed by Talluri and Aggarwal [85] by using feature correspondence between images taken by camera on robot and a CAD or similar model of its environment. For localization of car in urban environments, [86] used an inertial measurement unit and a sensor suite consists of four GPS antennas. Humanoid robots are getting popular as research tools as they offer new viewpoint compared to wheeled vehicle. A lot of work has been done so far on the localization for humanoid robots. In order to estimate the location of the robot [87] applied a vision based approach and then compared the current image to previously recorded reference images. In the local environment of the humanoid [88] detects objects with given colors and shapes and then determines its pose relative to these objects. With respect to a close object [89] localizes the robot to track the 6D pose of a manually initialized object relative to camera by applying a model based approach.

\section{Conclusion}

In this paper we reviewed MSN issues, sensor network applications in robotics and vice versa, robot localization, and also coverage for multiple robots. This is certainly not the extent that robotics is used in wireless sensor networks and also wireless sensor networks in robotics. However, we found that integrating static nodes with mobile robots enhances the capabilities of both types of devices and also enables new applications. The possibilities of robotics and wireless 
sensor networks being used together seem endless and if used together in future also will help to solve many problems.

\section{Conflict of Interests}

The authors declare no conflict of interests.

\section{Acknowledgment}

This work was supported by the Brain Korea 21 PLUS Project, National Research Foundation of Korea (NRF) grant funded by the Korean government (MEST) (no. 2013R1A2A2A01068127 and no. 2013R1A1A2A10009458).

\section{References}

[1] S. Basagni, A. Carosi, and C. Petrioli, "Mobility in wireless sensor networks," Journal of Wireless Networks, vol. 14, no. 6, pp. 831-858.

[2] C. Zhu, L. Shu, T. Hara, L. Wang, and S. Nishio, "Research issues on mobile sensor networks," in Proceedings of the 5th International ICST Conference on Communications and Networking in China (ChinaCom '10), pp. 1-6, IEEE, Beijing, China, August 2010.

[3] G. Song, Y. Zhou, F. Ding, and A. Song, "A mobile sensor network system for monitoring of unfriendly environments," Sensors, vol. 8, no. 11, pp. 7259-7274, 2008.

[4] http://www.wikipedia.com/.

[5] C. Flanagin, "A survey on robotics system and performance analysis," http://www.cse.wustl.edu/ jain/cse567-11/ftp/robots/.

[6] M. A. Goodrich and A. C. Schultz, "Human-robot interaction: a survey," Foundations and Trends in Human-Computer Interaction, vol. 1, no. 3, pp. 203-275, 2007.

[7] J. Reich and E. Sklar, "Robot-sensor Networks for search and rescue," in Proceedings of the IEEE International Workshop on Safety, Security and Rescue Robotics, Gaithersburg, Md, USA, August 2006.

[8] F. Amigoni, G. Fontana, and S. Mazzuca, "Robotic sensor networks: an application to monitoring electro-magnetic fields," in Proceedings of the 2007 Conference on Emerging Artificial Intelligence Applications in Computer Engineering, pp. 384-393, 2007.

[9] S. Shue and J. M. Conrad, "A survey of robotic applications in wireless sensor networks," in Proceedings of the IEEE Southeastcon, pp. 1-5, Jacksonville, Fla, USA, April 2013.

[10] http://en.wikipedia.org/wiki/Sensor_node.

[11] http://www.merriam-webster.com/dictionary/sensor.

[12] http://webs.cs.berkeley.edu/tos/.

[13] http://www.pages.drexel.edu/ kws23/tutorials/motes/motes .html.

[14] E. Stavrou and A. Pitsillides, "Security evaluation methodology for intrusion recovery protocols in wireless sensor networks," in Proceedings of the 15th ACM International Conference on Modeling, Analysis and Simulation of Wireless and Mobile Systems, pp. 167-170, Paphos, Cyprus, 2012.

[15] S. Meguerdichian, F. Koushanfar, M. Potkonjak, and M. B. Srivastava, "Coverage problems in wireless ad-hoc sensor networks," in Proceedings of the 20th Annual Joint Conference of the IEEE Computer and Communications Societies (INFOCOM '01), vol. 3, pp. 1380-1387, April 2001.
[16] B. Liu, O. Dousse, P. Nain, and D. Towsley, "Dynamic coverage of mobile sensor networks," IEEE Transactions on Parallel and Distributed Systems, vol. 24, no. 2, pp. 301-311, 2013.

[17] J. Luo and Q. Zhang, "Probabilistic coverage map for mobile sensor networks," in Proceedings of the IEEE Global Telecommunications Conference (GLOBECOM '08), pp. 1-5, New Orleans, La, USA, December 2008.

[18] O. Khatib, "Real-time obstacle avoidance for manipulators and mobile robots," in Proceedings of the IEEE International Conference on Robotics and Automation, vol. 2, IEEE, 1985.

[19] R. C. Arkin, "Motor schema-based mobile robot navigation," The International Journal of Robotics Research, vol. 8, no. 4, pp. 92-112, 1989.

[20] A. Howard, M. J. Matarić, and G. S. Sukhatme, "Mobile sensor network deployment using potential fields: a distributed, scalable solution to the area coverage problem," in Distributed Autonomous Robotic Systems 5, chapter 8, pp. 299308, Springer, Tokyo, Japan, 2002.

[21] S. Poduri and G. S. Sukhatme, "Constrained coverage for mobile sensor networks," in Proceedings of the IEEE International Conference on Robotics and Automation (ICRA '04), vol. 1, pp. 165-171, IEEE, May 2004.

[22] A. Howard, M. J. Mataríc, and G. S. Sukhatme, "An incremental self-deployment algorithm for mobile sensor networks," Autonomous Robots, vol. 13, no. 2, pp. 113-126, 2002.

[23] G. Wang, G. Cao, and T. F. La Porta, "Movement-assisted sensor deployment," IEEE Transactions on Mobile Computing, vol. 5, no. 6, pp. 640-652, 2006.

[24] G. Wang, G. Cao, and T. la Porta, "Movement-assisted sensor deployment," in Proceedings of the 23rd Annual Joint Conference of the IEEE Computer and Communications Societies (INFOCOM '04), pp. 2469-2479, March 2004.

[25] Y. Zou and K. Chakrabarty, "Sensor deployment and target localization based on virtual forces," in Proceedings of the 22nd Annual Joint Conference on the IEEE Computer and Communications Societies, pp. 1293-1303, April 2003.

[26] M. A. Batalin, M. Rahimi, Y. Yu et al., "Call and response: experiments in sampling the environment," in Proceedings of the 2nd International Conference on Embedded Networked Sensor Systems (SenSys '04), pp. 25-38, 2004.

[27] B. Liu, P. Brass, and O. Doussie, "Mobility improves coverage of sensor networks," in Proceedings of the 6th International Symposium on Mobile adhoc Networking (MobiHoc '05), pp. 300-308, 2005.

[28] A. Maxim and G. S. Batalin, "Coverage, exploration and deployment by a mobile robot and a communication network," in Proceedings of International Workshop on Information Processing in Sensor Networks, pp. 376-391, PaloAlto Research Center (PARC), Palo Alto, Calif, USA, April 2003.

[29] G. Wang, G. Cao, T. La Porta, and W. Zhang, "Sensor relocation in mobile sensor networks," in Proceedings of the 24th Annual Joint Conference of the IEEE Computer and Communications Societies (INFOCOM '05), vol. 4, pp. 2302-2312, Miami, Fla, USA, March 2005.

[30] I. Amundson and X. D. Koutsoukos, "Mobile sensor localization and navigation using RF doppler shifts," in Mobile Entity Localization and Tracking in GPS-less Environnments: Second International Workshop, MELT 2009, Orlando, FL, USA, September 30, 2009. Proceedings, vol. 5801 of Lecture Notes in Computer Science, pp. 235-254, Springer, Berlin, Germany, 2009. 
[31] Y. Ganggang and Y. Fengqi, "A localization algorithm for mobile wireless sensor networks," in Proceedings of the IEEE International Conference on Integration Technology (ICIT '07), pp. 623-627, March 2007.

[32] J. Yi, J. Koo, and H. Cha, "A localization technique for mobile sensor networks using archived anchor information," in Proceedings of the 5th Annual IEEE Communications Society Conference on Sensor, Mesh and Ad Hoc Communications and Networks (SECON '08), pp. 64-72, San Francisco, Calif, USA, June 2008.

[33] L. Hu and D. Evans, "Localization for mobile sensor networks," in Proceedings of the 10th Annual International Conference on Mobile Computing and Networking (MobiCom '04), pp. 45-57, October 2004.

[34] B. Dil, S. Dulman, and P. Havinga, "Range-based localization in mobile sensor networks," in Wireless Sensor Networks: Third European Workshop, EWSN 2006, Zurich, Switzerland, February 13-15, 2006. Proceedings, vol. 3868 of Lecture Notes in Computer Science, pp. 164-179, Springer, Berlin, Germany, 2006.

[35] S. Tilak, V. Kolar, N. B. Abu-Ghazaleh, and K.-D. Kang, "Dynamic localization control for mobile sensor networks," in Proceedings of the 24th IEEE International Performance, Computing, and Communications Conference (IPCCC '05), pp. 587-592, IEEE, April 2005.

[36] S. Tilak, V. Kolar, N. B. Abu Ghazaleh, and K.-D. Kang, "Dynamic localization protocols for mobile sensor networks," http://arxiv.org/abs/cs/0408042.

[37] B. Sau, S. Mukhopadhyaya, and K. Mukhopadhyaya, "Localization control to locate mobile sensors," in Distributed Computing and Internet Technology: Proceedings of the 3rd International Conference (ICDCIT '06), Bhubaneswar, India, December 20-23, 2006, vol. 4317 of Lecture Notes in Computer Science, pp. 81-88, Springer, Berlin, Germany, 2006.

[38] C. Saad, A. R. Benslimane, and J.-C. Koing, "A distributed method to localization for mobile sensor networks," in Proceedings of the IEEE Wireless Communications and Networking Conference (WCNC '07), 2007.

[39] http://en.wikipedia.org/wiki/Positioning_technology.

[40] http://en.wikipedia.org/wiki/ZigBee.

[41] Z. Farid, R. Nordin, and M. Ismail, "Recent advances in wireless indoor localization techniques and system," Journal of Computer Networks and Communications, vol. 2013, Article ID 185138, 12 pages, 2013.

[42] A. Popleteev, V. Osmani, and O. Mayora, "Investigation of indoor localization with ambient FM radio stations," in Proceedings of PerCom-2012, Lugano, Switzerland, March 2012.

[43] http://compnetworking.about.com/od/networkprotocols/g/ultra_wide_band.htm.

[44] C. Frost, C. S. Jensen, K. S. Luckow, B. Thomsen, and R. Hansen, "Bluetooth indoor positioning system using fingerprinting," in Mobile Lightweight Wireless Systems, vol. 81 of Lecture Notes of the Institute for Computer Sciences, Social Informatics and Telecommunications Engineering, pp. 136-150, Springer, Berlin, Germany, 2012.

[45] http://en.wikipedia.org/wiki/Hybrid_positioning_system.

[46] L. Niu, "A survey of wireless indoor positioning technology for fire emergency routing," IOP Conference Series: Earth and Environmental Science, vol. 18, Article ID 012127, 2014, Proceedings of the 8th International Symposium of the Digital Earth (ISDE '08).
[47] A. Cheriet, M. Ouslim, and K. Aizi, "Localization in a wireless sensor network based on RSSI and a decision tree," Przeglad Elektrotechniczny, vol. 89, no. 12, pp. 121-125, 2013.

[48] Y.-T. Chen, C.-L. Yang, Y.-K. Chang, and C.-P. Chu, "A RSSIbased algorithm for indoor localization using zigbee in wireless sensor network," in Proceedings of the 15th International Conference on Distributed Multimedia Systems (DMS '09), pp. 70-75, 2009.

[49] U. Ahmad, A. Gavrilov, U. Nasir, M. Iqbal, S. J. Cho, and S. Lee, "In-building localization using neural networks," in Proceedings of the IEEE International Conference on Engineering of Intelligent Systems (ICEIS '06), April 2006.

[50] L. Yu, "Fingerprinting localization based on neural networks and ultra wideband signals," in Proceedings of the IEEE International Symposium on Signal Processing and Information Technology (ISSPIT '11), pp. 184-189, 2011.

[51] C. Laoudias, D. G. Eliades, P. Kemppi, C. G. Panayiotou, and M. M. Polycarpou, "Indoor localization using neural networks with location fingerprints," in Artificial Neural Networks-ICANN 2009, vol. 5769 of Lecture Notes in Computer Science, pp. 954963, Springer, Berlin, Germany, 2009.

[52] C. Nerguizian, C. Despins, and S. Affès, "Indoor geolocation with received signal strength fingerprinting technique and neural networks ," in Telecommunications and NetworkingICT 2004: 11th International Conference on Telecommunications, Fortaleza, Brazil, August 1-6, 2004. Proceedings, vol. 3124 of Lecture Notes in Computer Science, pp. 866-875, Springer, Berlin, Germany, 2004.

[53] S. Kumar and S.-R. Lee, "Localization with RSSI values for wireless sensor networks: an artificial neural network approach," in Proceedings of the International Electronic Conference on Sensors and Applications, 2014, Paper d007.

[54] S.-H. Fang and T.-N. Lin, "Indoor location system based on discriminant-adaptive neural network in IEEE 802.11 environments," IEEE Transactions on Neural Networks, vol. 19, no. 11, pp. 1973-1978, 2008.

[55] D. Cobzas and H. Zhang, "Cylindrical panoramic image-based model for robot localization," in Proceedings of the IEEE/RSJ International Conference on Intelligent Robots and Systems (IROS '01), pp. 1924-1930, November 2001.

[56] B. J. A. Kröse, N. Vlassis, and R. Bunschoten, "Omni directional vision for appearance-based robot localization," in Sensor Based Intelligent Robots, vol. 2238 of Lecture Notes in Computer Science, pp. 39-50, Springer, 2002.

[57] N. Aida Mahiddin, E. Nadia Madi, S. Dhalila, E. Fadzli Hasan, S. Safie, and N. Safie, "User position detection in an indoor environment," International Journal of Multimedia and Ubiquitous Engineering, vol. 8, no. 5, pp. 303-312, 2013.

[58] J. Xu, W. Liu, F. Lang, Y. Zhang, and C. Wang, "Distance measurement model based on RSSI in WSN," Wireless Sensor Network, vol. 2, pp. 606-611, 2010.

[59] G. Jekabsons, V. Kairish, and V. Zuravlyov, "An analysis of WiFi based indoor positioning accuracy," Scientific Journal of Riga Technical University, Computer Sciences, vol. 44, no. 1, pp. 131137, 2012.

[60] S. Capkun, M. Hamdi, and J. P. Hubaux, "GPS free positioning in mobile ad hoc networks," Journal Cluster Computing, vol. 5, no. 2, pp. 157-167, 2002.

[61] D. Djenouri, L. Khelladi, and N. Badache, "A survey of security issues in mobile ad hoc and sensor networks," IEEE Communications Surveys and Tutorials, vol. 7, no. 4, pp. 2-28, 2005. 
[62] Y. Wang, G. Attebury, and B. Ramamurthy, "A survey of security issues in wireless sensor networks," IEEE Communications Surveys \& Tutorials, vol. 8, no. 2, pp. 2-23, 2006.

[63] V. Kumar, A. Jain, and P. N. Barwa, "Wireless sensor networks: security issues, challenges and solutions," International Journal of Information and Computation Technology, vol. 4, no. 8, pp. 859-868, 2014.

[64] H. Kaur, "Attacks in wireless sensor networks," Research Cell. In press.

[65] Y.-C. Hu, A. Perrig, and D. B. Johnson, "Wormhole attacks in wireless networks," IEEE Journal on Selected Areas in Communications, vol. 24, no. 2, pp. 370-380, 2006.

[66] G. Padmavathi and D. Shanmugapriya, "A survey of attacks, security mechanisms and challenges in WSN," International Journal of Computer Science and Information Security, vol. 4, no. 1-2, 2009.

[67] A. LaMarca, W. Brunette, D. Koizumi et al., "PlantCare: an investigation in practical ubiquitous systems," in UbiComp 2002: Ubiquitous Computing: 4th International Conference Göteborg, Sweden, September 29-October 1, 2002 Proceedings, vol. 2498 of Lecture Notes in Computer Science, pp. 316-332, Springer, Berlin, Germany, 2002.

[68] X. Li, I. Lille, R. Falcon, A. Nayak, and I. Stojmenovic, "Servicing wireless sensor networks by mobile robots," IEEE Communications Magazine, vol. 50, no. 7, pp. 147-154, 2012.

[69] S. M. Schaffert, Closing the loop: control and robot navigation in wireless sensor networks [Ph.D. thesis], EECS Department, University of California, Berkeley, Calif, USA, 2006.

[70] J.-P. Sheu, K.-Y. Hsieh, and P.-W. Cheng, "Design and implementation of mobile robot for nodes replacement in wireless sensor networks," Journal of Information Science and Engineering, vol. 24, no. 2, pp. 393-410, 2008.

[71] J. Reich and E. Sklar, "Robotic sensor networks for search and rescue," in Proceedings of the IEEE International Workshop on Safety, Security, and Rescue Robotics (SSRR '06), 2006.

[72] F. Amigoni, G. Fontana, and S. Mazzuca, "Robotic sensor networks: an application to monitoring electro-magnetic fields," in Proceedings of the Conference on Emerging Artificial Intelligence Applications in Computer Engineering: Real Word AI Systems with Applications in eHealth, HCI, Information Retrieval and Pervasive Technologies, pp. 384-393, IOS Press, Amsterdam, The Netherlands, 2007.

[73] L. Iocchi, D. Nardi, and M. Salerno, "Reactivity and deliberation: a survey on multi-robot systems," in Balancing Reactivity and Social Deliberation in Multi-Agent Systems, vol. 2103 of Lecture Notes in Computer Science, pp. 9-32, Springer, Berlin, Germany, 2001.

[74] B. Walenz, "Multi robot coverage and exploration: a survey of existing techniques," http://bwalenz.files.wordpress.com/2010/ 06/csci8486-walenz-paper.pdf.

[75] S. K. Chan, A. P. New, and I. Rekleitis, "Distributed coverage with multi-robot system," in Proceedings of the IEEE International Conference on Robotics and Automation (ICRA '06), pp. 2423-2429, Orlando, Fla, USA, May 2006.

[76] M. A. Batalin and G. S. Sukhatme, "Spreading out: a local approach to multi-robot coverage," in Proceedings of the 6th International Symposium on Distributed Autonomous Robotics System, pp. 373-382, Fukuoka, Japan, June 2002.

[77] B. Ranjbar-Sahraei, G. Weiss, and A. Nakisaee, "stigmergic coverage algorithm for multi-robot systems (demonstration)," in Proceedings of the 10th German conference (MATES '12), pp. 126-138, Springer, Trier, Germany, October 2012.
[78] I. A. Wagner, M. Lindenbaum, and A. M. Bruckstein, "Distributed covering by ant-robots using evaporating traces," IEEE Transactions on Robotics and Automation, vol. 15, no. 5, pp. 918933, 1999.

[79] N. Hazon and G. A. Kaminka, "On redundancy, efficiency, and robustness in coverage for multiple robots," Robotics and Autonomous Systems, vol. 56, no. 12, pp. 1102-1114, 2008.

[80] E. Royer, M. Lhuillier, M. Dhome, and J.-M. Lavest, "Monocular vision for mobile robot localization and autonomous navigation," International Journal of Computer Vision, vol. 74, no. 3, pp. 237-260, 2007.

[81] R. G. Brown and B. R. Donald, "Mobile robot self-localization without explicit landmarks," Algorithmica, vol. 26, no. 3-4, pp. 515-559, 2000.

[82] V. Varveropoulos, Robot Localization and Map construction using sonar data, The Rossum project.

[83] F. Dellaert, D. Fox, W. Burgard, and S. Thrun, "Robust Monte Carlo localization for mobile robots," Artificial Intelligence, vol. 128, no. 1-2, pp. 99-141, 2001.

[84] F. Dellaert, W. Burgard, D. Fox, and S. Thrun, "Using the condensation algorithm for robust, vision-based mobile robot localization," in Proceedings of the IEEE Computer Society Conference on Computer Vision and Pattern Recognition (CVPR '99), pp. 588-594, June 1999.

[85] R. Talluri and J. K. Aggarwal, "Mobile robot self-location using model-image feature correspondence," IEEE Transactions on Robotics and Automation, vol. 12, no. 1, pp. 63-77, 1996.

[86] R. Nayak, "Reliable and continuous urban navigation using multiple Gps antenna and a low cost IMU," in Proceedings of the ION GPS, Salt Lake City, Utah, USA, September 2000.

[87] J. Ido, Y. Shimizu, Y. Matsumoto, and T. Ogasawara, "Indoor navigation for a humanoid robot using a view sequence," International Journal of Robotics Research, vol. 28, no. 2, pp. 315325, 2009.

[88] R. Cupec, G. Schmidt, and O. Lorch, "Experiments in visionguided robot walking in a structured scenario," in Proceedings of the IEEE International Symposium on Industrial Electronics (ISIE '05), pp. 1581-1586, Dubrovnik, Croatia, June 2005.

[89] P. Michel, J. Chestnutt, S. Kagami, K. Nishiwaki, J. Kuffner, and T. Kanade, "GPU-accelerated real-time 3D tracking for humanoid locomotion and stair climbing," in Proceedings of the IEEE/RSJ International Conference on Intelligent Robots and Systems (IROS '07), pp. 463-469, IEEE, San Diego, Calif, USA, November 2007. 

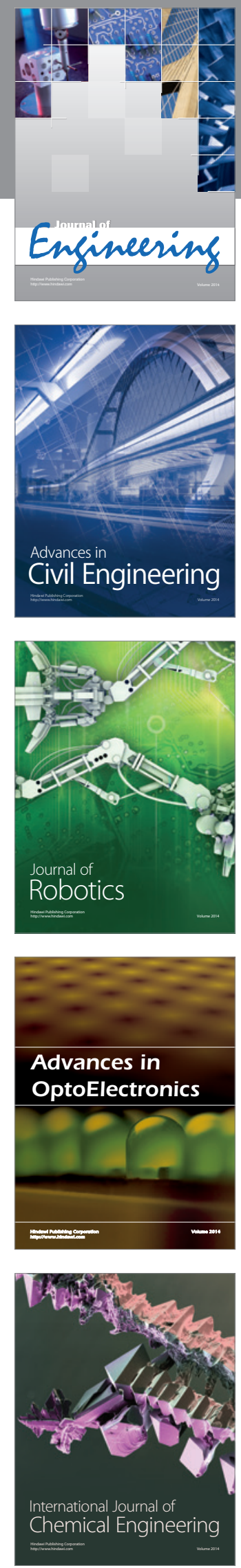

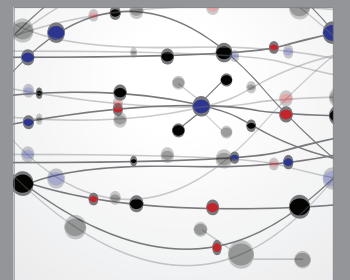

The Scientific World Journal
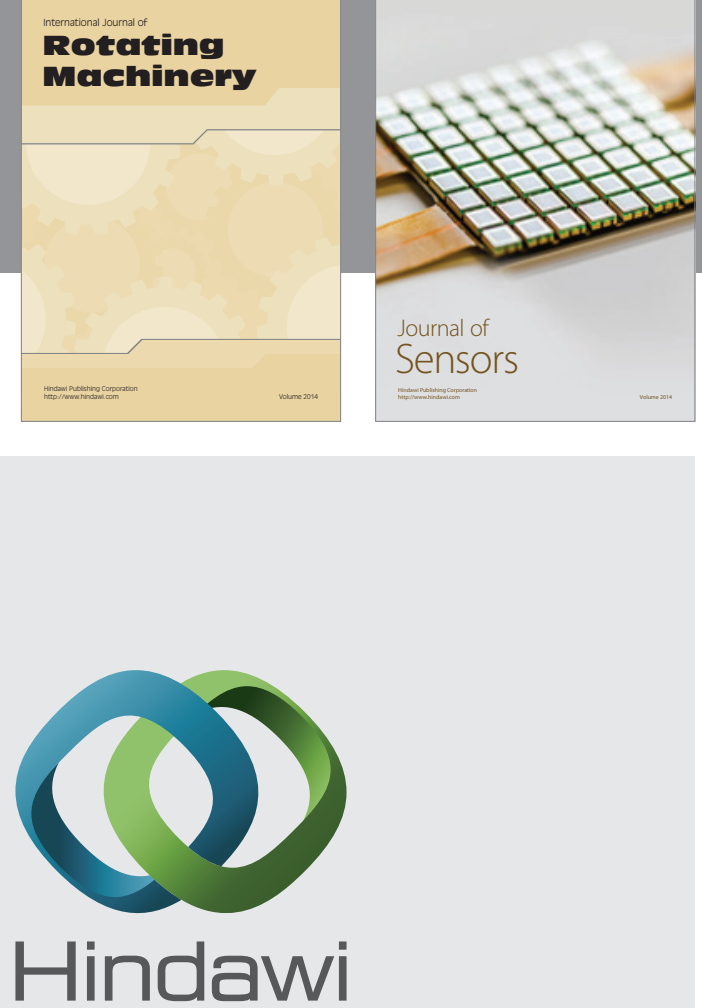

Submit your manuscripts at http://www.hindawi.com
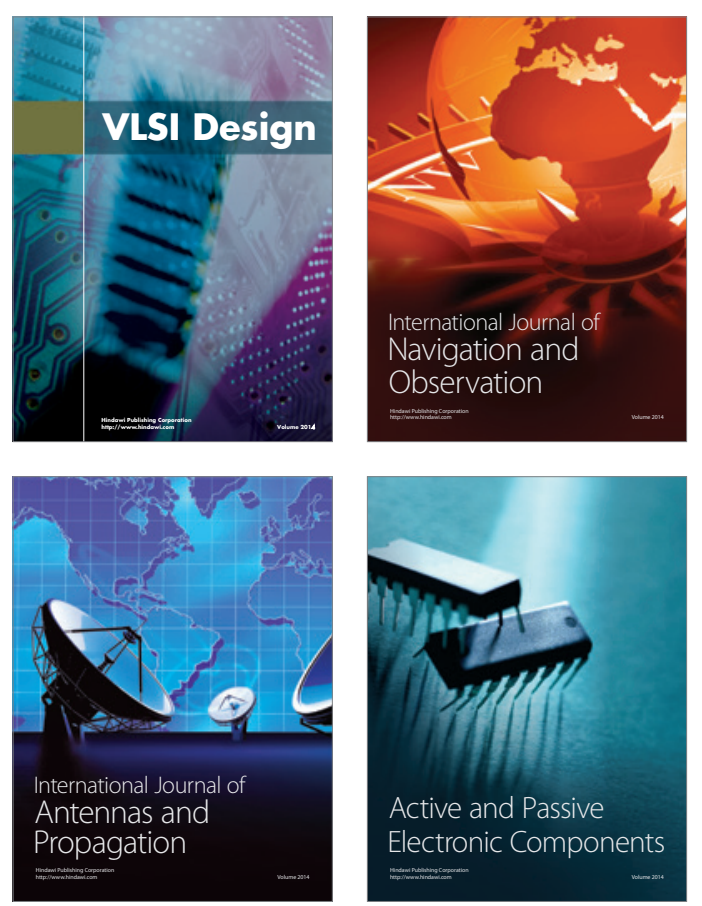
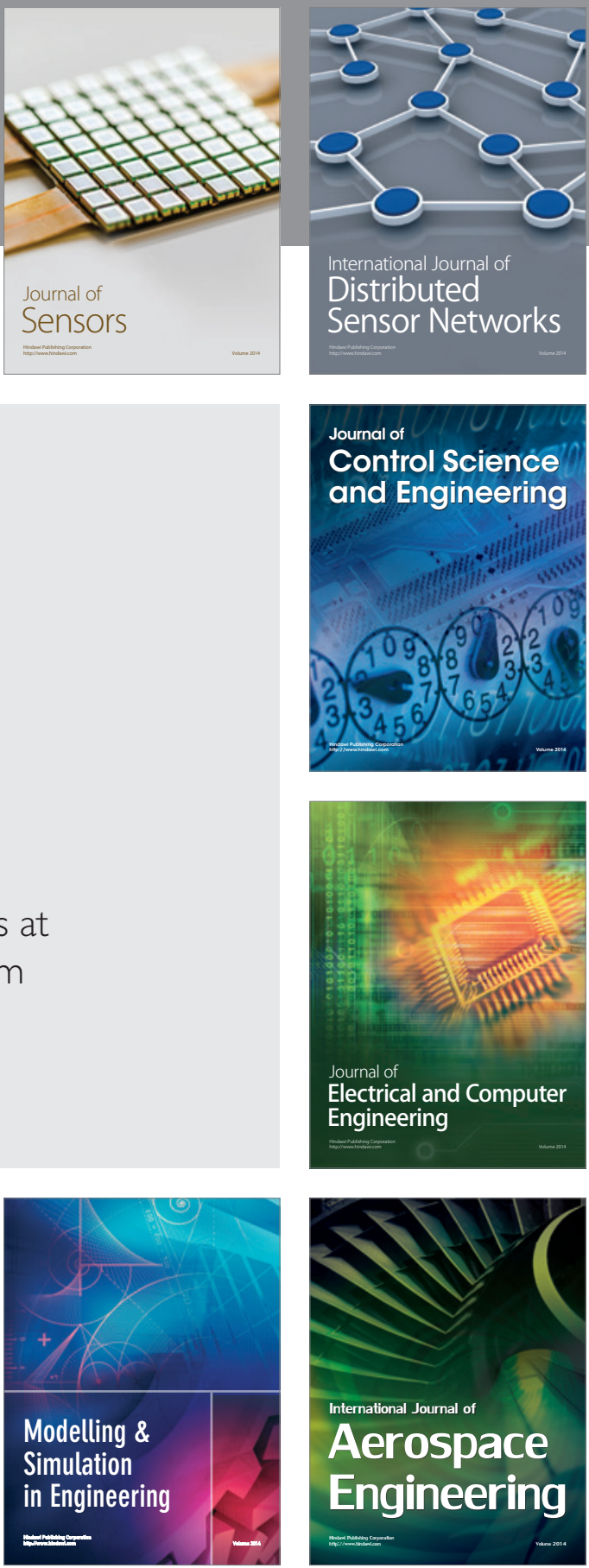

Journal of

Control Science

and Engineering
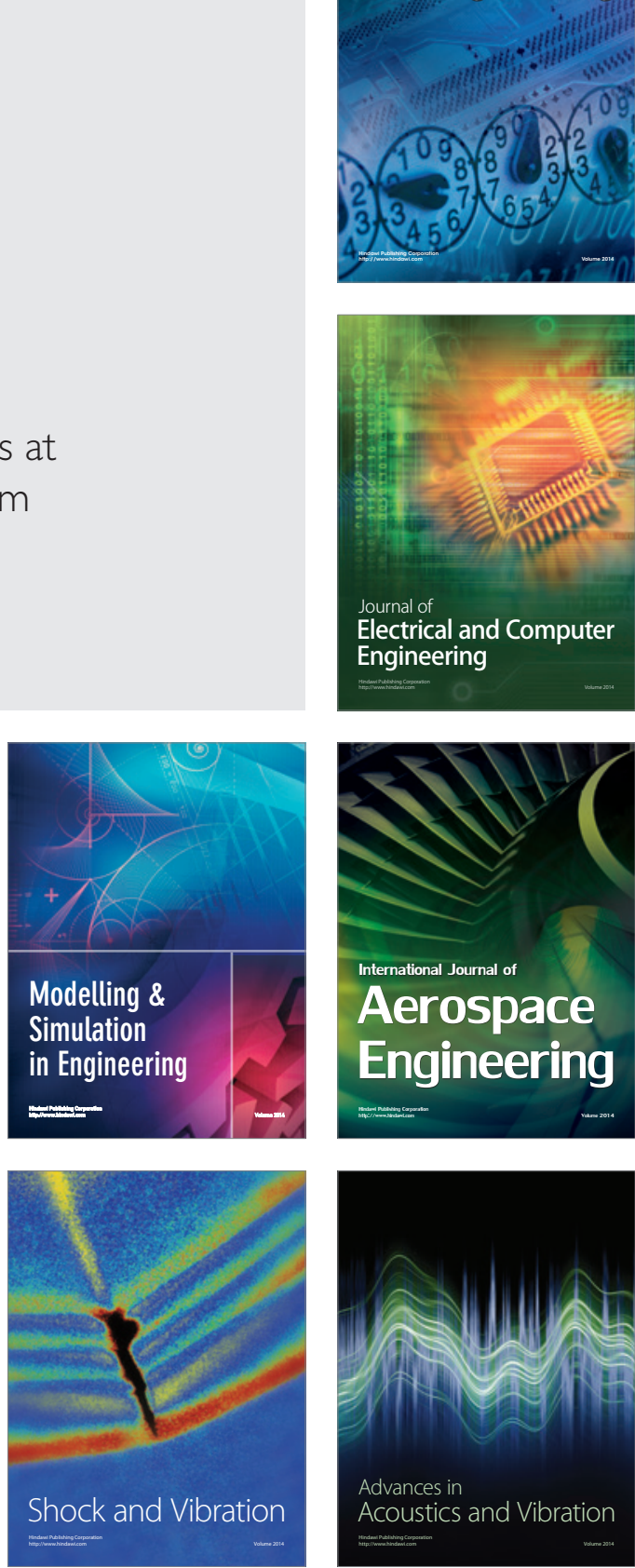